\title{
Human carriage of Yersinia spp.
}

\author{
MELODY GREENWOOD and W. L. HOOPER
}

\section{Public Health Laboratory, Poole General Hospital, Poole, Dorset BH15 2JB}

\begin{abstract}
Summary. Examination of faecal specimens for the presence of Yersinia spp. during a 1 -year period yielded isolations from $3.5 \%$ of patients. Salmonella spp., Campylobacter spp. and Shigella spp. were isolated from $2.5 \%, 2.8 \%$ and $0.34 \%$ of patients respectively. Most isolates belonged to $Y$. enterocolitica biotype $1(53 \%)$ and $Y$. frederikseni $(39 \%)$. The most frequently encountered serotypable strains were serotypes O 5,27 and O 6,30. Serotype O 3, the commonly recognised pathogenic strain, was not isolated in this survey. A strong association between Yersinia excretion and the age group 1-14 years was demonstrated. Although biotype-1 strains and $Y$. frederikseni have not previously been thought to be pathogenic, clinical significance could be attributed to the presence of Yersinia spp. in almost $90 \%$ of patients aged 15 years or more, and in over $50 \%$ of patients in the younger age groups.
\end{abstract}

\section{Introduction}

During the last 20 years, Yersinia enterocolitica has become accepted as a causative agent of bacterial gastro-enteritis, terminal ileitis and mesenteric adenitis (Bottone, 1977; Lancet, 1984). Infection may also lead to several auto-immune conditions, in particular erythema nodosum and polyarthritis (Aho et al., 1981; Winblad, 1981). The organisms may further be responsible for a wide variety of other clinical manifestations (Larson, 1979). Sporadic infection has been reported in many countries (Swaminathan et al., 1982) and several outbreaks of infection due to $Y$.enterocolitica have also been recorded (Asakawa et al., 1973; Black et al., 1978; Ratnam et al., 1982; Shayegani et al., 1983; Tacket et al., 1984). Despite the increasing number of reports of $Y$. enterocolitica isolations elsewhere, there is little published information concerning the incidence of this organism in Great Britain. Between 1980 and 1982, only 213 reports of patients with $Y$. enterocolitica infection were received by the Communicable Diseases Surveillance Centre (Public Health Laboratory Service, personal communication) of which 122 isolates were of faecal origin. Almost all of the strains which had been serotyped belonged to serotype $\mathrm{O} 3$, which is the common pathogenic strain in this country.

To determine carriage rates in the local community, faecal samples submitted to the laboratory during a 1-year period were examined for the

Received 16 May 1986; accepted 13 Sep. 1986. presence of Yersinia spp. The results obtained are presented.

\section{Materials and methods}

Faecal specimens and rectal swabs submitted to the laboratory for conventional bacteriological examination from hospital and general practice patients were also cultured for the presence of $Y$. enterocolitica and related organisms. Specimens were applied directly to the surface of Cefsulodin-Irgasan-Novobiocin (CIN) agar plates (Yersinia Selective Agar, Oxoid CM653, with Oxoid antibiotic supplement SR109). Specimens were also inoculated into $10 \mathrm{ml}$ of buffered peptone water (BPW; Oxoid CM509) containing peptone $1 \%$; this was incubated at $4^{\circ} \mathrm{C}$ for $17-21$ days before subculture to CIN agar. All CIN agar plates were incubated at $30^{\circ} \mathrm{C}$ for 20 $24 \mathrm{~h}$. Suspect colonies were identified by methods described elsewhere (Greenwood and Hooper, 1985). Briefly, isolates were screened with Triple Sugar Iron (TSI) agar (Oxoid) and urea broth. Those organisms that produced an acid butt in TSI without gas or $\mathrm{H}_{2} \mathrm{~S}$ production and were urease-positive were further tested for absence of motility at $37^{\circ} \mathrm{C}$. Presumptive strains of Yersinia were sent to the reference facility at Leicester Public Health Laboratory for serotyping and biotyping according to the methods of Wauters (1970). Serum samples obtained from patients excreting Yersinia organisms were also sent to Leicester for antibody detection.

\section{Results}

During the 1-year period, 4585 faecal specimens or rectal swabs from 3784 patients were examined. A total of 135 strains of Yersinia spp. was isolated from 133 patients, representing a patient isolation rate of $3.5 \%$. Corresponding isolation rates for 
Salmonella spp., Campylobacter spp. and Shigella spp. were $2 \cdot 5 \%, 2 \cdot 8 \%$ and $0.34 \%$ respectively. Only five strains of Yersinia were isolated by direct culture; all other strains were obtained after cold enrichment. Species, biotypes and serotypes of the Yersinia strains are shown in tables I and II. Most isolates belonged to $Y$. enterocolitica biotype 1 or $Y$. frederikseni. Serotypes O 5,27 and O 6,30 occurred most frequently. Serotype O 3, the most commonly recognised pathogenic strain, was not isolated.

Table I. Species and biotypes of Yersinia isolated from human faeces

\begin{tabular}{lc}
\hline Species & Number of isolates \\
\hline Y. enterocolitica biotype 1 & 72 \\
Y. enterocolitica biotype 3 & 5 \\
Y. frederikseni & 51 \\
Y. intermedia & 4 \\
Y.pseudotuberculosis & 1 \\
Not typed & 2 \\
\hline
\end{tabular}

Table II. Serotypes of Yersinia spp. isolated from human faeces

\begin{tabular}{lcc}
\hline Species (biotype) & O serotype & Number of isolates \\
\hline Y. enterocolitica (1) & 5,27 & 23 \\
Y. enterocolitica (3) & 5,27 & 1 \\
Y. enterocolitica (1) & 6,30 & 13 \\
Y. enterocolitica (1) & 7 & 6 \\
Y. enterocolitica (1) & 15 & 2 \\
Y. enterocolitica (1) & 34 & 1 \\
Y. enterocolitica (1) & NT & 26 \\
Y. enterocolitica (3) & NT & 4 \\
Y. frederikseni & 16 & 9 \\
Y. frederikseni & NT & 43 \\
Y. intermedia & NT & 4 \\
Y.pseudotuberculosis & IIa & 1 \\
Not typed & - & 2 \\
\hline
\end{tabular}

NT = not typable.

Analysis of patients by age and sex is shown in table III. Excretion was strongly associated with age $\left(x^{2}=42 \cdot 8 ; \mathrm{p}<0 \cdot 001\right)$. Isolations in the $1-4$ and 5-14 age groups were twice those expected. Although Yersinia excretion occurred more often in females aged 1-24 years, there was no overall association with $\operatorname{sex}\left(x^{2}=6 \cdot 32 ; p>0 \cdot 5\right)$. Analysis of the occurrence of gastro-intestinal symptoms in relation to age is shown in table IV. Of patients from whom no other pathogen was isolated, $65 \%$ experienced symptoms of diarrhoea, vomiting,
Table III. Analysis of patients excreting Yersinia spp. by age and sex

\begin{tabular}{lrrrrr}
\hline & \multicolumn{3}{c}{ Number of patients } & \multicolumn{2}{c}{$\begin{array}{c}\text { Percentage } \\
\text { of }\end{array}$} \\
\cline { 2 - 6 } Age (years) & male & female & not stated & total & $\begin{array}{c}\text { pa- } \\
\text { tients }\end{array}$ \\
\hline$<1$ & 5 & 2 & 1 & 8 & $6 \cdot 0$ \\
$1-4$ & 15 & 27 & - & 42 & $31 \cdot 6$ \\
$5-14$ & 7 & 10 & - & 17 & $12 \cdot 8$ \\
$15-24$ & 7 & 12 & - & 19 & $14 \cdot 3$ \\
$25-34$ & 6 & 3 & - & 9 & $6 \cdot 8$ \\
$35-44$ & 5 & 2 & - & 7 & $5 \cdot 3$ \\
$45-54$ & 3 & 3 & - & 6 & $4 \cdot 5$ \\
$55-64$ & 6 & 1 & - & 7 & $5 \cdot 3$ \\
$>65$ & 5 & 6 & - & 11 & $8 \cdot 3$ \\
Not stated & 4 & 3 & - & 7 & $5 \cdot 3$ \\
Total & 63 & 69 & 1 & 133 & 100 \\
& & & & & \\
\hline
\end{tabular}

Table IV. Occurrence of gastro-intestinal symptoms in relation to age

\begin{tabular}{lcccc}
\hline & \multicolumn{2}{c}{ Symptoms* } & & \\
\cline { 2 - 3 } Age & $+(\%)$ & $-(\%)$ & $\begin{array}{c}\text { Not } \\
\text { known }\end{array}$ & $\begin{array}{c}\text { Other } \\
\text { pathogens }\end{array}$ \\
\hline$<1$ & $4(50)$ & $4(50)$ & 0 & 0 \\
$1-4$ & $23(59)$ & $16(31)$ & 2 & 3 \\
$5-14$ & $6(46)$ & $7(54)$ & 1 & 1 \\
$>15$ & $52(88)$ & $7(12)$ & 0 & 6 \\
Not known & 1 & 0 & 0 & 0 \\
Total & $85(71)$ & $34(29)$ & 3 & 10 \\
\hline
\end{tabular}

* Symptoms : + = diarrhoea, vomiting, loose stools or abdominal pain $;-=$ no gastro-intestinal symptoms.

loose stools or abdominal pain. Most other isolates were made on routine testing of faeces from children admitted to hospital for other reasons (31), nursing staff (3) or people returning from foreign travel. Blood samples were obtained from 10 patients 310 weeks after the faecal samples were submitted, but no serum antibody response to Yersinia spp. could be demonstrated.

\section{Discussion}

The incidence of yersinia infection rivals that of salmonella infection in some surveys (Marks et al., 1980; Weissfeld and Sonnenwirth, 1980), but appears to be low in other reports (Dajani and Maurer, 1980; Yamauchi et al., 1981). The excretion of Yersinia revealed in this survey exceeds that of Salmonella, Campylobacter and Shigella spp. Before the introduction of CIN agar the isolation of $Y$. 
enterocolitica from faecal material relied heavily on the use of media designed for the detection of other enteric pathogens. The differences in isolation rate obtained by other workers are probably due to differing isolation techniques rather than to different geographical location.

$Y$. enterocolitica biotype 1 and $Y$. frederikseni were the predominant groups of Yersinia spp. isolated. The isolation of $Y$. frederikseni from clinical sources has not been reported before in this country (Public Health Laboratory Service, personal communication), possibly as a result of the use of unsuitable isolation media, but perhaps also because this organism has only recently been identified amongst the so-called " $Y$. enterocoliticalike" organisms (Bercovier et al., 1980; Brenner et al., 1980, Ursing et al., 1980). This investigation has shown that serotype $\mathrm{O} 3$ is not the most common type of Yersinia spp. to be found in human faecal specimens in the UK. Serotype O 3 was not isolated during this 1-year study, although subsequent studies in which the methodology described here was used have resulted in several isolations of this serotype.

The pathogenicity of serotypes $\mathrm{O} 3, \mathrm{O} 9$ and $\mathrm{O} 8$ is well established, but opinion varies concerning the pathogenicity of other serotypes and of strains such as Y. frederikseni. Van Noyen et al. (1981) found that strains of Yersinia other than serotypes O 3 and O 9 were recovered only by use of cold enrichment techniques. These workers did not consider such strains to be significant as they were twice as prevalent in controls as in patients. Weissfeld and Sonnenwirth (1980) found only biotype-1 strains in a survey of patients in hospital, and felt that all their isolates were of clinical significance. They found an incidence rate of $Y$. enterocolitica equal to that of Salmonella and emphasised the need for cold enrichment. Martin et al. (1982) also stressed the need for cold enrichment methods, and showed that a biotype-1 strain of serotype 21 was pathogenic. Pai et al. (1979) found that although recovery of serotype O 3 from symptomatic patients was usually obtained by direct culture, recovery of other serotypes was very poor without cold enrichment. These workers also found that recovery of serotype $\mathrm{O} 3$ from the stools

\section{REFERENCES}

Aho K, Ahvonen P, Laitinen O, Leirisalo M 1981 Arthritis associated with Yersinia enterocolitica. In: Bottone E J (ed) Yersinia enterocolitica, CRC Press Inc., Boca Raton, FL, pp 113-124. of asymptomatic carriers and convalescent patients was considerably enhanced by the use of cold enrichment. Results obtained in our study confirm the need for cold enrichment; less than $5 \%$ of isolations were made by direct culture.

Results shown in tables III and IV indicate that yersinia carriage is strongly associated with the 114 year age group. However, this group also contains the largest proportion of apparently symptomless excreters. It has been shown (Greenwood and Hooper, 1985) that pasteurised milk often contains Yersinia organisms. The greatest consumption of milk occurs in this age group and may account partly for this frequency of isolation. If results obtained from patients less than 15 years of age are excluded, it can be seen that almost $90 \%$ of excreters exhibit gastro-intestinal symptoms. It seems unlikely that the finding of Yersinia serotypes other than those of the recognised pathogenic strains in such a large proportion of symptomatic patients is merely fortuitous. In a review of $Y$. frederikseni diarrhoea (Scholey and Freeman, 1984), the authors speculated that the organism might be a cause of antibiotic-associated diarrhoea. Other clinical manifestations of yersiniosis may also include pharyngitis (Tacket et al., 1983) fever, arthritis and erythema nodosum (Bottone, 1977) which may occur in the absence of, or following, gastrointestinal symptoms. The availability of clinical information in this survey was limited to the details given on the request forms, and follow-up of positive cases was hampered by the time lapse involved in cold enrichment methods.

The regimen combining enrichment in BPW incubated at $4^{\circ} \mathrm{C}$ for $17-21$ days with subculture to CIN agar has allowed a more complete picture of the incidence of Yersinia spp. in man to emerge. Studies are in progress in an effort to reduce the length of time required to isolate Yersinia spp. from enrichment media. This will allow better follow-up of clinical cases and help to clarify the role of all strains of Yersinia in enteric disease.

We thank Mr B. Ford and his staff for technical assistance, and the reference facility at Leicester Public Health Laboratory for biotyping and serotyping of Yersinia strains and for testing for serum antibodies.
Asakawa Y, Akahane S, Kagata N, Noguchi M, Sakazaki R, Tamura K 1973 Two community outbreaks of human infection with Yersinia enterocolitica. Journal of Hygiene 71 : 715-723.

Bercovier $\mathrm{H}$ et al. 1980. Yersinia kristensenii: a new species of Enterobacteriaceae composed of sucrose-negative strains 
(formerly called atypical Yersinia enterocolitica or Yersinia enterocolitica-like). Current Microbiology 4: 219-224.

Black R E et al. 1978 Epidemic Yersinia enterocolitica infection due to contaminated chocolate milk. New England Journal of Medicine 298: 76-79.

Bottone E J 1977 Yersinia enterocolitica: a panoramic view of a charismatic microorganism. CRC Critical Reviews in Microbiology 5 : 211-241.

Brenner D J et al. 1980 Yersinia intermedia: a new species of Enterobacteriaceae composed of rhamnose-positive, melibiose-positive, raffinose-positive strains (formerly called Yersinia enterocolitica or Yersinia enterocolitica-like). Current Microbiology 4: 207-212.

Dajani A S, Maurer M J 1980 Is Yersinia enterocolitica gastroenteritis a Canadian disease? Journal of Pediatrics 97 : 165.

Greenwood M H, Hooper W L 1985 Yersinia spp. in foods and related environments. Food Microbiology 2: 263-269.

Lancet 1984 Yersiniosis today. Lancet 1: 84-85.

Larson J H 1979 The spectrum of clinical manifestations of infection with Yersinia enterocolitica and their pathogenesis. In: Carter P B et al. (eds) Yersinia enterocolitica: biology, epidemiology and pathology (Contributions to microbiology and immunology vol 5). Karger, Basel pp 257-269.

Marks M I, Pai C H, Lafleur L, Lackman L, Hammerberg O 1980 Yersinia enterocolitica gastroenteritis: a prospective study of clinical, bacteriologic and epidemiologic features. Journal of Pediatrics 96: 26-31.

Martin T, Kasian G F, Stead S 1982 Family outbreak of yersiniosis. Journal of Clinical Microbiology 16: 622-626.

Pai C H, Sorger S, Lafleur L, Marks M I 1979 Efficacy of cold enrichment techniques for recovery of Yersinia enterocolitica from human stools. Journal of Clinical Microbiology 9: 712715.

Ratnam S, Mercer E, Picco B, Parsons S and Butler R 1982 A nosocomial outbreak of diarrhoeal disease due to Yersinia enterocolitica serotype O:5, biotype 1. Journal of Infectious Diseases 145: 242-247.
Scholey J, Freeman H J 1984 Yersinia frederiksenii [diarrhea:] review of a one-year experience with a new inflammatory bowel disease syndrome. Gastroenterology 86: 1237.

Shayegani M, Morse D, DeForge I, Root T, Parsons L M, Maupin P S 1983 Microbiology of a major foodborne outbreak of gastroenteritis caused by Yersinia enterocolitica serogroup 0:8. Journal of Clinical Microbiology 17: 35-40.

Swaminathan B, Harmon M C, Mehlman I J 1982 A review: Yersinia enterocolitica. Journal of Applied Bacteriology 52 : 151-183.

Tacket C O, Davis B R, Carter G P, Randolph J F, Cohen M L 1983 Yersinia enterocolitica pharyngitis. Annals of Internal Medicine 99 : 40-42.

Tacket C O et al. 1984 A multi-state outbreak of infections caused by Yersinia enterocolitica transmitted by pasteurized milk. Journal of the American Medical Association 251 : 483 486.

Ursing J et al. 1980 Yersinia frederiksenii: a new species of Enterobacteriaceae composed of rhamnose-positive strains (formerly called atypical Yersinia enterocolitica or Yersinia enterocolitica-like). Current Microbiology 4: 213-217.

Van Noyen R, Vandepitte J, Wauters G, Selderslaghs R 1981 Yersinia enterocolitica: its isolation by cold enrichment from patients and healthy subjects. Journal of Clinical Pathology 34: 1052-1056.

Wauters G 1970 Contribution à l'étude de Yersinia enterocolitica. Vander, Louvain, Belgium.

Weissfeld A S, Sonnenwirth A C 1980 Yersinia enterocolitica in adults with gastrointestinal disturbances: need for cold enrichment. Journal of Clinical Microbiology 11 : 196-197.

Winblad S 1981 Erythema nodosum associated with infection with Yersinia enterocolitica. In: Bottone E J (ed) Yersinia enterocolitica. CRC Press Inc., Boca Raton, FL, pp 125133.

Yamauchi T, Scroggin C, Euler A, Eisenach K 1981 Incidence of Yersinia and Campylobacter from stool cultures in the South Central United States. Abstracts of the Annual Meeting of the American Society of Microbiology, Abstract no. C218, p 299. 